\title{
Reactive versus Latching Phase Control of a Two-body Heaving Wave Energy Converter
}

\author{
António F. de O. Falcão, Paulo A. P. Justino, João C. C. Henriques and José M. C. S. André
}

\begin{abstract}
The paper deals with the modelling, optimization and control of the IPS buoy wave energy converter, a two-body point-absorber oscillating in heave, consisting of a floater rigidly connected to a long submerged vertical acceleration tube open at both ends within which a piston can slide, forcing the motion of the inside water below and above it. Energy is converted from the relative motion between the piston and the buoy-tube pair. The modelling is performed in the frequency domain (including reactive phase control), in order to provide some kind of geometry optimization, and also in the timedomain to allow the simulation of a more realistic highpressure-oil power take-off system.
\end{abstract}

\section{INTRODUCTION}

The concept of point absorber for wave energy utilization was developed in the late 1970s and early 1980s, mostly in Scandinavia. This is in general a wave energy converter of oscillating body type whose horizontal dimensions are small compared to the representative wave length. In its simplest version, the body reacts against the sea bottom. In deep water (say 50-m depth or more), this may raise difficulties due to the distance between the floating body and the bottom. Multi-body systems may then be used instead, in which the energy is converted from the relative motion between two bodies oscillating differently.

One of the most interesting two-body point absorbers for wave energy conversion is the IPS buoy, invented by Sven A. Noren [1] and initially developed in Sweden by the company Interproject Service (IPS). A half-scale prototype of the IPS buoy was tested in sea trials in Sweden, in the early 1980s [2]. The AquaBuOY is a wave energy converter, developed in the 2000s, that combines the IPS buoy concept with a pair of hose pumps to produce a flow of water at high pressure that drives a Pelton turbine [3]. A prototype of the AquaBuOY was deployed and tested in 2007 in the Pacific Ocean off the coast of Oregon.

The theoretical dynamics of a two-body heaving wave energy converter has been analysed in detail by Falnes [4] (see also [5-7]). Here, we consider a simplified version of the IPS buoy. Results from an optimization study in the frequency domain (linear power take-off) are presented for regular as well as irregular waves, including the effect of

Manuscript received October 11, 2008. This work was supported in part by IDMEC Lisbon, through the Portuguese Foundation for Science and Technolgy (program POCI).

A. Falcão (corresponding author, phone: 351-21-8417273, fax: 351-218417398, e-mail: antonio.falcao@ist.utl.pt), J. Henriques (e-mail: joaochenriques@ist.utl.pt) and J. André (e-mail: jmandre@hidro1.ist.utl.pt) are with IDMEC, Instituto Superior Técnico, Universidade Técnica de Lisboa, 1049-001 Lisbon, Portugal.

P. Justino is with Instituto Nacional de Engenharia, Tecnologia e Inovação, 1649-038 Lisbon, Portugal. (e-mail paulo.justino@mail.ineti.pt). reactive phase control. Numerical results are then presented from a time-domain study for a more realistic high-pressureoil power take-off system (PTO).

\section{THEORETICAL MODEL}

\subsection{Basic assumptions}

The IPS buoy consists basically of a buoy rigidly connected to a submerged tube (the acceleration tube), oscillating in heave, by the action of the waves, with respect to a piston that can slide along the tube. The wave energy is absorbed by means of the relative motion between the piston and the buoy-tube set. The concept is represented in Fig. 1a.
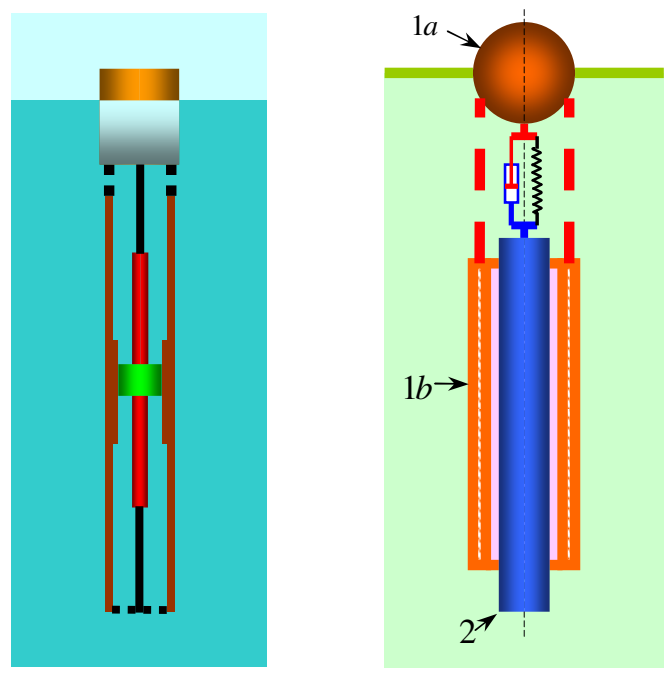

Fig. 1. (a) On the left, the IPS buoy; (b) on the right, a simplified representation.

We note that most of the inertia against which the buoy moves is that of the water contained inside the acceleration tube (obviously in addition to the mass of the piston itself). In the simplified mathematical modelling presented in this section, the IPS buoy is replaced by two bodies oscillating independently in heave (Fig. 1b). Body 1 consists of a floater (body $1 a$, shown as a semi-submerged sphere, a geometry adopted here for modelling) rigidly connected to a fully submerged body (body $1 b$, that represents the inertia of the acceleration tube walls). We neglect the mass and volume of the structure linking bodies $1 a$ and $1 b$ (or include it in the mass of body $1 b$ ), as well as the hydrodynamic forces on the structure. Body 2 represents the inertia of the piston plus the water inside the tube. In the frequency domain analysis, we will assume that bodies 1 and 2 are connected by a linear damper-spring PTO, as shown in Fig. 1b.

Because the converter performance depends on body geometry as well as on PTO parameters and control strategy 
and algorithm, the optimization is not an easy task. To render it more feasible, we introduce some simplifying assumptions.

Firstly, we fix the shape and size of the floating body $1 a$. Then we assume that the distance from the submerged bodies $1 b$ and 2 to the free surface is large enough so that (i) they are subject neither to excitation forces nor to radiation forces, and (ii) their added masses are independent of frequency of oscillation. Besides, we neglect the hydrodynamic interaction between bodies $1 a, 1 b$ and 2 .

\subsection{Basic equations in the frequency domain}

We consider the two-body system represented in Fig. 1b, and assume that both bodies are constrained to oscillate in heave, a reasonable approximation taking into account the relatively large length of the submerged tube. Let $x$ and $y$ be the coordinates for the heaving motion of bodies 1 and 2 , respectively, with $x=y=0$ at equilibrium and $x, y$ increasing upwards.

The equations of motion can be found in [8] for an arbitrary number of oscillating bodies, and in [4] for the specific case of two bodies oscillating in heave. We may write, for the motion of bodies 1 and 2, acted upon by sinusoidal waves of frequency $\omega$,

$\left(m_{1}+A_{1}\right) \ddot{x}+B \dot{x}+\rho g S x+C(\dot{x}-\dot{y})+K(x-y)=f_{d}$,

$\left(m_{2}+A_{2}\right) \ddot{y}-C(\dot{x}-\dot{y})-K(x-y)=0$.

Here $\rho$ is water density, $g$ is acceleration of gravity, $m_{j}$ ( $j=1$ for body $1, j=2$ for body 2 ) is body mass, $A_{j}(\omega)$ is added mass, $B(\omega)$ is radiation damping coefficient (of body 1a), $S$ is the cross sectional area of body 1a defined by the undisturbed free-surface, $C$ is the damping coefficient due to the power take-off mechanism and $K$ is the stiffness of the spring. Finally $f_{d}$ is the hydrodynamic excitation force on body $1 \mathrm{a}$.

If the whole system is linear (which requires the damper and the spring to be linear), we may write $x(t)=X_{0} e^{i \omega t}$, $y(t)=Y_{0} e^{i \omega t}$, where $X_{0}$ and $Y_{0}$ are (in general complex) amplitudes, and $f_{d}(t)=A_{w} \Gamma(\omega) e^{i \omega t}$. Here $A_{w}$ is the incident wave (real) amplitude, and $\Gamma$ is the (in general complex) excitation (or diffraction) force coefficient for body 1a. From $(1,2)$, we obtain

$$
\begin{aligned}
& X_{0}\left(-\omega^{2}\left(m_{1 a}+A_{1 a}(\omega)+M_{1 b}\right)+i \omega(B+C)+\rho g S\right) \\
& \quad-Y_{0}(i \omega C+K)=A_{w} \Gamma, \\
& X_{0}(-i \omega C-K)+Y_{0}\left(-\omega^{2} M_{2}+i \omega C+K\right)=0 .
\end{aligned}
$$

Here $M_{1 b}=m_{1 b}+A_{1 b}$ and $M_{2}=m_{2}+A_{2}$ are the mass plus added mass of bodies $1 b$ (acceleration tube) and 2 (water in tube plus piston), respectively. Note that $A_{1 b}$ and $A_{2}$ are supposed not to be functions of frequency $\omega$ (as a consequence from the assumption of deep submergence). The complex amplitudes $X_{0}$ and $Y_{0}$ can easily be obtained by solving the system of equations $(3,4)$.
The time-averaged power extracted from the waves is given by $\bar{P}=\omega^{2} C\left|X_{0}-Y_{0}\right|^{2} / 2$.

We assume the floating body $1 a$ to be a hemisphere. Tabulated values (together with asymptotic expressions) can be found in [9] (in dimensionless form) for the added mass $A_{1 a}(\omega)$ and the radiation resistance coefficient $B(\omega)$ of body $1 a$ deep water. The absolute value of the excitation force coefficient $\Gamma(\omega)$ may be obtained from $B(\omega)$ by using the Haskind relation (valid for an axisymmetric body oscillating in heave, see [8])

$|\Gamma(\omega)|=\left(\frac{2 g^{3} \rho B(\omega)}{\omega^{3}}\right)^{1 / 2}$.

Since we only consider heave oscillations, the equations of motion are not affected by how the mass $m_{1}=m_{1 a}+m_{1 b}$ is distributed between bodies $1 a$ and $1 b$. For convenience of presentation of numerical results, we assume that $m_{1 a}$ is the mass of water of volume equal to the submerged part of the buoy in the absence of waves (in the present case a hemisphere).

\section{NUMERICAL RESULTS FOR LINEAR PTO}

We adopt the simplified model, in deep water, as described in sub-section 2.2 and shown in Fig. 1b, with a floating hemispherical buoy of diameter $15 \mathrm{~m}$, and assume a linear PTO. We set $g=9.8 \mathrm{~ms}^{-2}, \rho=1025 \mathrm{~kg} \mathrm{~m}^{-3}$, and define dimensionless values denoted by an asterisk. For mass, it is $M_{1 b}^{*}=M_{1 b} / m_{1 a}$ and $M_{2}^{*}=M_{2} / m_{1 a}$, where $m_{1 a}=905.7 \times 10^{3} \mathrm{~kg}$. We also define dimensionless values for PTO damping and stiffness as $C^{*}=C / B\left(\omega_{8}\right)$ and $K^{*}=K /(\rho g S), \quad$ respectively. Here it is $\rho g S=1.776 \mathrm{MN} \mathrm{m}^{-1}, \omega_{8}=2 \pi / 8 \mathrm{rad} / \mathrm{s}$ is the frequency for wave period $T=8 \mathrm{~s}$, and $B\left(\omega_{8}\right)=242.5 \mathrm{kN} \mathrm{m}^{-1} \mathrm{~s}$. Finally, we define the dimensionless absorbed power as $\bar{P}^{*}=\bar{P} / \bar{P}_{\max }$. Here $\bar{P}_{\max }$ is the theoretical maximum limit of the (time-averaged) power that an axisymmetric heaving wave energy converter can absorb from regular waves of frequency $\omega$ and amplitude $A_{w}$, and is known to be (see e.g. [8]) $\bar{P}_{\max }=g^{3} \rho A_{w}^{2} /\left(4 \omega^{3}\right)$ (corresponding to capture width $\lambda / 2 \pi$ ).

For irregular waves, a Pierson-Moskowitz spectral distribution was adopted, defined by (SI units, see [10])

$S(\omega)=526 H_{s}^{2} T_{e}^{-4} \omega^{-5} \exp \left(-1054 T e^{-4} \omega^{-4}\right)$,

where $H_{s}$ is significant wave height and $T_{e}$ is energy period. The time-averaged power output in irregular waves is computed as

$$
\bar{P}_{\text {irr }}\left(H_{s}, T_{e}\right)=\int_{0}^{\infty} \bar{P}_{1}(\omega) S(\omega) d \omega,
$$


where $\bar{P}_{1}(\omega)$ is the power absorbed from regular waves of frequency $\omega$ and unit amplitude. The time-averaged theoretical maximum power that can be absorbed from the irregular waves by an axisymmetric (one- or two-body) heaving converter is

$\bar{P}_{\text {max,irr }}=\frac{g^{3} \rho}{4} \int_{0}^{\infty} \omega^{-3} S(\omega) d \omega=149.5 H_{s}^{2} T_{e}^{3}$.

As for regular waves, we define $\bar{P}_{\text {irr }}^{*}=\bar{P}_{\text {irr }} / \bar{P}_{\text {max }, \text { irr }}$.
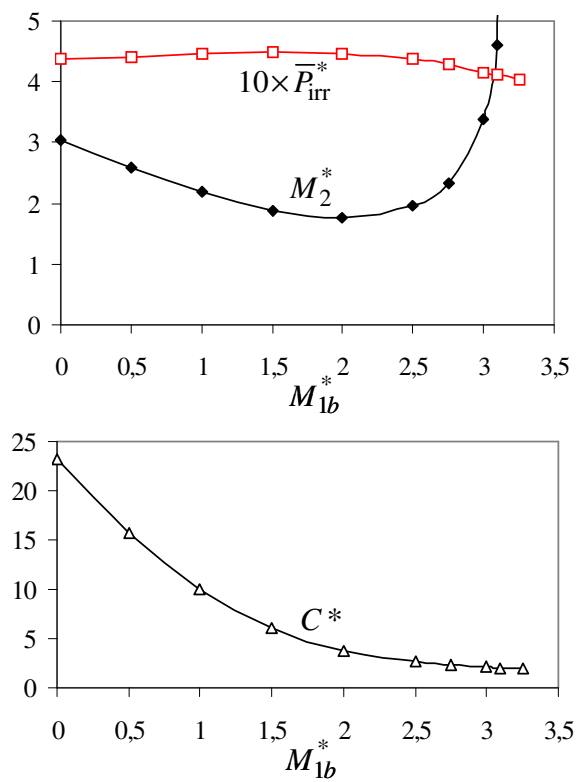

Fig. 2. Results from optimization in irregular waves versus $M_{1 b}^{*}$, for $T_{e}=8 \mathrm{~s}$ (linear damper, no spring).

Results were computed for $T_{e}=8 \mathrm{~s}$ and a PTO consisting of a linear damper of coefficient $C$ (no spring, $K=0$, i.e. non-reactive phase control). For each $M_{1 b}^{*}$, values of the pair $M_{2}^{*}, C^{*}$ were obtained that yield maximum $\bar{P}_{\text {irr }}^{*}$. The results of this optimisation are plotted versus $M_{1 b}^{*}$ in Fig. 2, together with $10 \times \bar{P}_{\text {irr }}^{*}$.

Unlike in the case of regular waves, it is not longer possible to extract the theoretical maximum power from irregular waves with a linear damper as PTO. In the simulated case, the power that can be absorbed can be seen to be weakly dependent on the ratio $M_{1 b} / m_{1 a}$ and equal to about $41-44 \%$. The curve for $M_{2}^{*}$ exhibits a minimum. To the left of this point, we have the "strong damping" solution (characterized by small mass $M_{1 b}^{*}$ and small relative motion amplitude); to the right is the "week damping" solution (large $M_{1 b}^{*}$, in the limit $M_{1 b}^{*}=\infty$ i.e. a single body converter).

\subsection{Phase control}

It is known that optimal (non-linear) phase control would allow the theoretical maximum to be attained (i.e. $\bar{P}_{\text {irr }}^{*}=1$ ).
Unfortunately optimal phase control is unfeasible, for well known reasons [11]: it would require the prediction of the incoming waves (and also relatively heavy computing that cannot be easily implemented in real time). Besides it would imply reactive control, i.e. the reversal of the energy flow direction during part of the cycle, with the negative consequences if the reactive power peaks are not small and (friction) losses are significant in the two-way energy transfer process (see [12]). An alternative control method that avoids the energy flow reversal was proposed by Budal and Falnes [13] and consists in latching the device in a fixed position (in the present case relative position of bodies 1 and 2) during certain intervals of the wave cycle (see also $[15,16]$ ). This will be considered in section 4.1 .

\subsection{Reactive phase control in irregular waves}

In this subsection we consider linear reactive control, simulated by a linear damper (coefficient $C$ ) and a linear spring (negative stiffness $K$ ).

In order to fix the masses $M_{1 b}$ and $M_{2}$, we make use of the optimized results shown in Fig. 2, and look for masses of bodies $1 b$ and 2 that are not unacceptably high. We choose $M_{1 b}=2 m_{1 a}, M_{2}=1.76 m_{1 a}$, a pair which, together with $C=3.77 B\left(\omega_{8}\right)$, was found to maximize the absorbed energy for $T_{e}=8 \mathrm{~s}$ with a non-reactive linear PTO. The following step is to simulate reactive control by allowing $K$ to take negative values and look for optimal values of $C$. The results are represented is Figs 3, 4 for $T_{e}=8$ and $12 \mathrm{~s}$, and show that, if $K$ is allowed to take unrestricted negative values, substantially more power can be absorbed, especially for the larger periods. For example, for $T_{e}=12 \mathrm{~s}, \bar{P}_{\text {irr }}^{*}$ attains $38 \%$ (for $K^{*}=-0.63$, Fig. 4 ), whereas the maximum is $12 \%$ if $K$ is constrained to be zero. Off course, large negative values of $K$ in reactive phase control are to be avoided in practice for the reasons mentioned above.

\section{TIME-DOMAIN MODELLING FOR NON-LINEAR PTO (HIGH PRESSURE OIL)}

\subsection{Latching phase-control}

It may be of interest to investigate how phase control by latching (rather then reactive phase control) can provide an increase in absorbed energy. Here we assume that latching is provided by a high-pressure-oil PTO, that includes a hydraulic ram, a hydraulic motor and a gas accumulator system (as in some wave energy converters like Pelamis and Wavebob). In the modelling of this PTO we closely follow [14]. In the case of a single body oscillating in heave, latching control was found to significantly increase the amount of absorbed energy by a relatively small ( $5 \mathrm{~m}$ radius hemispherical buoy) point absorber [14].

In the case of two bodies, by latching we mean that, during a part of the wave cycle (latched period), the two bodies are constrained to remain rigidly connected to each other; obviously, unlike for a single body system, latching does not 

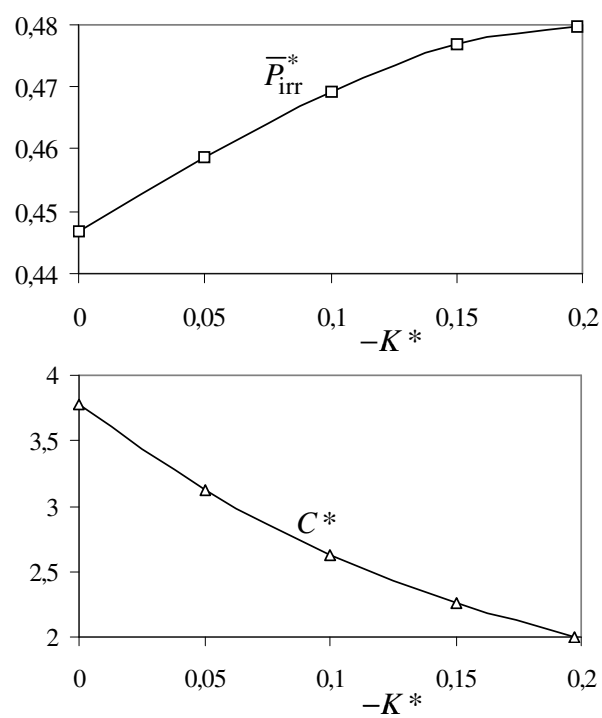

Fig. 3. Results from optimization (linear PTO) with reactive control versus negative spring stiffness, in irregular waves of $T_{e}=8 \mathrm{~s}$.
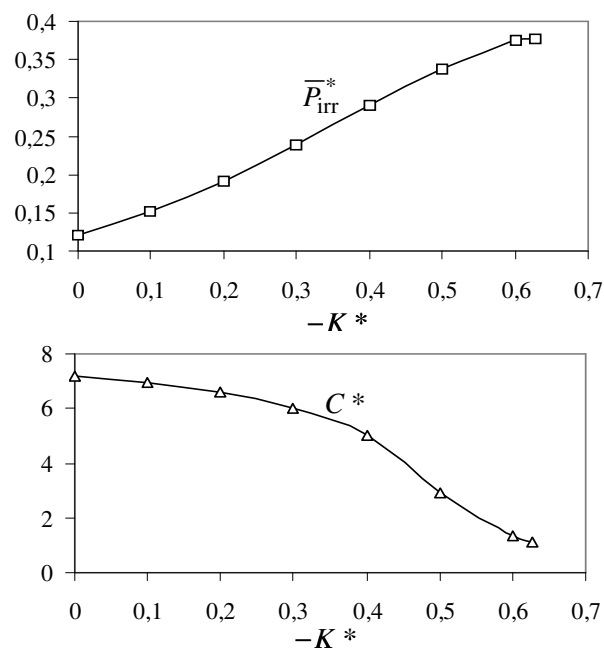

Fig. 4. As in Fig. 4, for $T_{e}=12 \mathrm{~s}$.

constrain any of the two bodies (including especially the energy extracting floating body) to remain motionless (with respect to the sea bottom).

Here we assume the relative motion between bodies 1 and 2 to drive a two-way hydraulic ram that feeds high pressure oil to a hydraulic motor. A gas accumulator system, consisting of a high-pressure (HP) reservoir and a low pressure (LP) reservoir, is placed in the circuit to produce a smoothing effect. Such a wave energy converter is highly non-linear, which requires a time-domain model consisting of a set of coupled equations: (i) a set of two differential equations (one of which constrains a convolution integral representing the radiation memory effect) that account for the hydrodynamics of the two-body wave energy absorber [8]; (ii) an ordinary differential equation that models the time-varying gas volume and pressure, the dependence of the oil flow rate (supplied to the hydraulic motor) on accumulator pressure, and the non-return valve system [14]. Standard methods were employed to numerically integrate the differential equations, with appropriate initial conditions (for details, see [14,17]). Instead of $(3,4)$, the governing equations for the hydrodynamics are now

$$
\begin{aligned}
& \left(m_{1 a}+A_{1 a}(\infty)+M_{1 b}\right) \ddot{x}(t)+\rho g S x(t) \\
& \quad+\int_{-\infty}^{t} L(t-\tau) \ddot{x}(t) d \tau-f_{d}(t)=f_{m}, \\
& M_{2} \ddot{y}(t)=-f_{m} .
\end{aligned}
$$

The memory function $L$ can be obtained from the radiation damping coefficient $B(\omega)$ (see [17]). In $(9,10), f_{m}$ is the vertical force on body 1 due to the PTO mechanism. This force will depend on the instantaneous gas pressures in the HP and LP accumulators and on the control algorithm.

We consider first the case in which the force $f_{m}$ is insufficient to move the piston inside the hydraulic ram (and so $\dot{x}=\dot{y}$ ) for as long as $\left|f_{m}\right|<S_{c} \Delta p$, where $S_{c}$ is the piston area and $\Delta p$ is the instantaneous value of the pressure difference between the HP and LP accumulators. This kind of damping (simple Coulomb damping) does not involve any phase control strategy and was analysed in detail (for a single body) in [17]. For this situation, the control algorithm proposed in [17] consists in establishing a proportionality relationship $q_{m}=S_{c}^{2} G \Delta p$ between the instantaneous liquidflow rate through the hydraulic motor, $q_{m}$, and $\Delta p$. Here $G$ is a control parameter. The power available to the hydraulic machine is $P_{m}=q_{m} \Delta p=G\left(S_{c} \Delta p\right)^{2}$. We note that, over a sufficiently long time, the time-averaged values of $P$ (power absorbed from the waves) and $P_{m}$ are equal (no energy losses are assumed to occur in the hydraulic circuit).

As in [14], this kind of hydraulic PTO can be used to achieve a phase control by extending the period of time during which bodies 1 and 2 remain fixed to each other. When the bodies are moving, their relative velocity, $\dot{x}-\dot{y}$, will, at some time, come to zero, as a result of the hydrodynamic forces on their wetted surfaces and the PTO forces. The bodies will then remain fixed to each other until force $\left|f_{m}\right|$ exceeds $R\left(S_{c} \Delta p\right)$, where $R>1$. It is to be noted that the force that has to be overcome (if the piston is to restart moving) is now larger (by a factor $R$ ) as compared with the simple Coulomb damping (i.e. compared with $\left.S_{c} \Delta p\right)$. There is now a new parameter, $R$, to be optimized, jointly with parameter $G$.

Numerical simulations ( $83 \mathrm{~min}$ long each) were carried out, based on this procedure and algorithm, for the two-body system (in deep water) represented in Fig. 1b, with a hemispheric floater radius $a=7.5 \mathrm{~m}$. Piston area (in hydraulic ram) was $S_{c}=0.0314 \mathrm{~m}^{2}$. The masses of gas (nitrogen) in the HP and LP accumulators were $200 \mathrm{~kg}$ and $40 \mathrm{~kg}$, respectively. The Pierson-Moskowitz spectrum (11) was discretized into 225 equally spaced $(\Delta \omega=0.01 \mathrm{rad} / \mathrm{s}$ ) sinusoidal harmonics in the range $0.25-2.5 \mathrm{rad} / \mathrm{s}$. The differential equations were numerically integrated in the time domain with a time step size of $0.02 \mathrm{~s}$. 
The results presented in Figs 5,6 were computed for $H_{s}=2 \mathrm{~m}$, and several values of dimensionless masses $M_{1 b}^{*}$ and $M_{2}^{*}$, time period $T_{e}$, and control parameters $G$ and $R$. The curves were plotted versus control parameter $G$ (for the oil flow rate through the hydraulic motor).
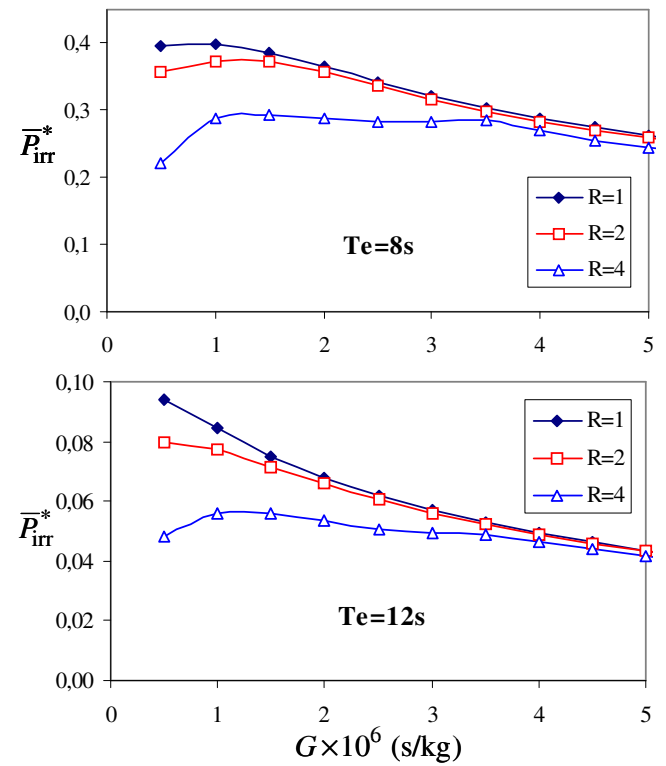

Fig. 5. Results from latching control with high-pressure-oil PTO, for masses $M_{1 b}^{*}=2$ and $M_{2}^{*}=1.76$, and several values of $T_{e}$ and control parameter $R$.

In Fig. 5, the same values $M_{1 b}^{*}=2$ and $M_{2}^{*}=1.76$ were adopted as in Figs 3-4 (where the PTO is linear). The maximum values of $\bar{P}_{\text {irr }}^{*}$ obtainable with the non-linear hydraulic PTO are slightly lower (by about $10 \%$ ) than the ones shown in Figs 3,4 for $K=0$ (no reactive phase control). It is to be noted that no improvement was found by increasing control parameter $R$ above unity (i.e. introducing latching), unlike the beneficial effect of negative spring stiffness (reactive phase control) observed in Figs 3-4 with a linear PTO. This seems to be due to the fact that the effect intended with latching (keeping the floating energyabsorbing body motionless during part of the wave cycle) in ineffective due to the finite mass of body 2 .

To further investigate this, numerical simulations were performed for a smaller mass of body $1 \mathrm{~b}\left(M_{1 b}^{*}=1\right)$ and increasingly larger masses of body $2: M_{2}^{*}=6$ (Fig. 6) and $M_{2}^{*}=\infty$ (Fig. 7). Latching (i.e. control parameter $R>1$ ) was found to be marginally effective for $M_{2}^{*}=6$ and the long wave period $T_{e}=14 \mathrm{~s}$. As expected, the improvement from latching may be substantial (especially for the larger wave periods) for $M_{2}^{*}=\infty$, i.e. a single body reacting against the sea bottom, as shown in Fig. 7 .

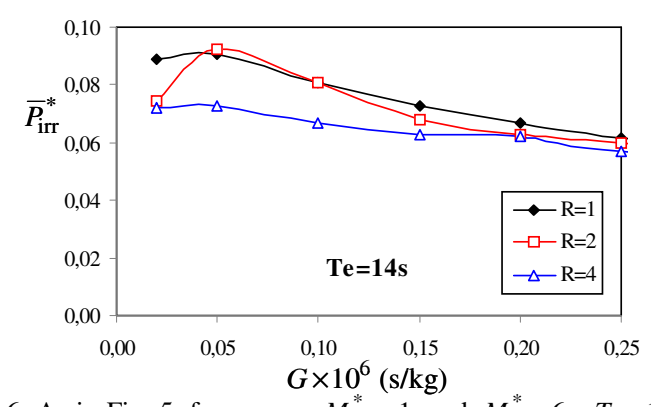

Fig. 6. As in Fig. 5, for masses $M_{1 b}^{*}=1$ and $M_{2}^{*}=6, T_{e}=14 \mathrm{~s}$ and several values of control parameter $R$.

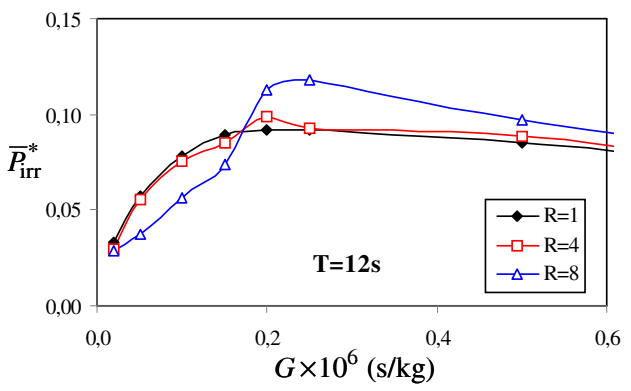

Fig. 7. As in Fig. 5, for masses $M_{1 b}^{*}=1$ and $M_{2}^{*}=\infty, T_{e}=12 \mathrm{~s}$ and several values of control parameter $R$.
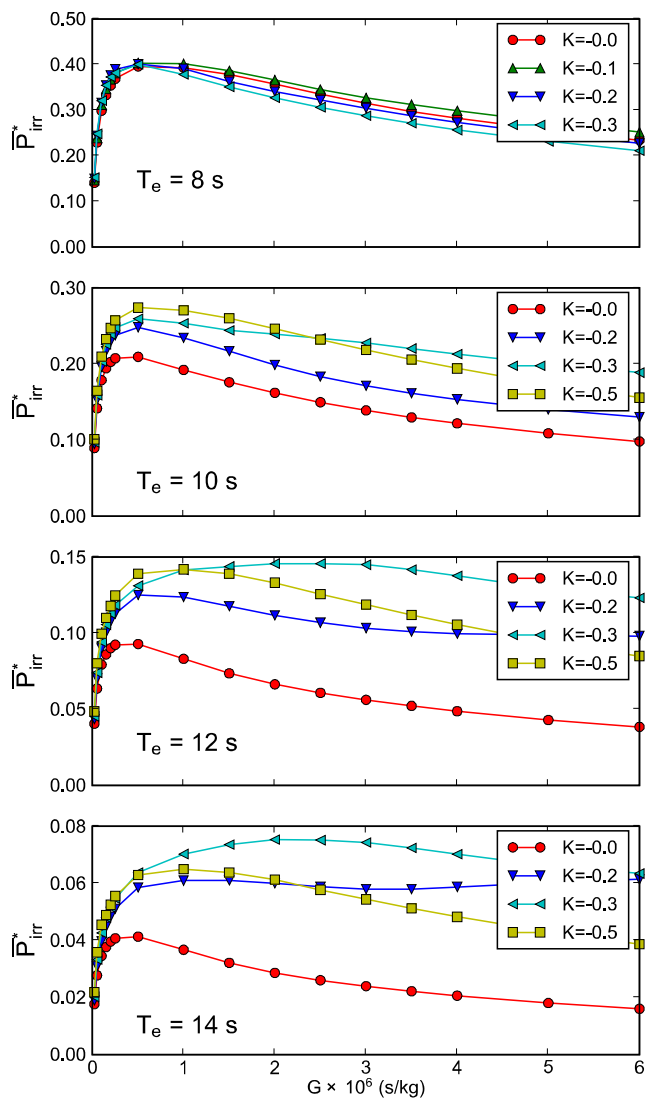

Fig. 8. Results from a PTO combining a hydraulic circuit (flow control parameter $G$ ) with a negative spring, for masses $M_{1 b}^{*}=2$ and $M_{2}^{*}=1.76$, and several values of $T_{e}$ and non-dimensional spring stiffness $K^{*}$. 


\subsection{Reactive phase control}

In principle, it seems possible to achieve reactive phasecontrol with a hydraulic PTO as the one outlined here, by suitably reversing the direction of the oil flow (and delivering energy to the surrounding wave field) during part of the wave cycle, as a means of increasing the overall absorbed energy. Numerical simulations (not reported here) showed this not to be effective.

An additional simulation was performed for a PTO consisting of a high-pressure-oil circuit (as above, with $R=1$ ) combined (not very realistically) with a linear "spring" of negative stiffness $K$, providing reactive phase control. Numerical results, shown in Fig. 8, illustrate the effectiveness of such a PTO, especially for the longer wave periods. However, as could be anticipated, this requires large "negative-spring" forces $|K(x-y)|$ which most of the time greatly exceed what the be provided by the hydraulic circuit (i.e largely exceed $S_{c} \Delta p$ ) (see Fig. 9). This indicates that effective reactive control requires larger forces (and larger exchanges of energy) than what can be provided by the PTO systems usually proposed for wave energy converters.

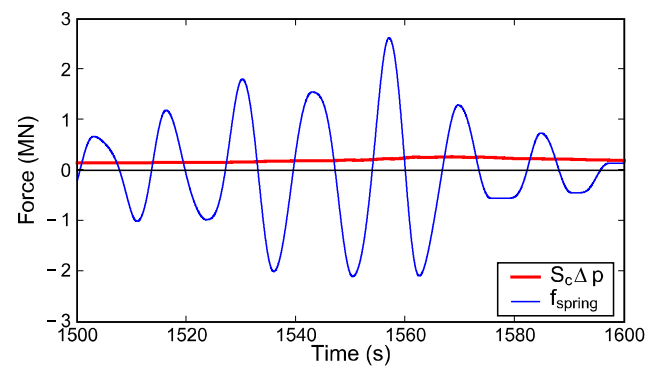

Fig. 8. Comparison between force on piston $S_{c} \Delta p$ and negativespring (reactive) force $K(x-y)$, for $M_{1 b}^{*}=2, M_{2}^{*}=1.76$, $T_{e}=12 \mathrm{~s}, \quad K^{*}=-0.3$ and $G=2 \times 10^{-6} \mathrm{~s} / \mathrm{kg}$.

\section{Conclusions}

Simulations were performed, in the frequency domain (regular and irregular waves), for a simplified version of the IPS buoy (a two-body wave energy converter) equipped with a linear damper as PTO.

It was found that, by introducing a "spring" with negative stiffness (reactive phase control), the amount of absorbed energy (from irregular waves) can be significantly increased, especially for the longer wave periods.

The substantial increase in absorbed energy by latching phase-control of a single-body converter (known from the published literature) was found not to occur for the two-body IPS buoy under consideration, except for (possibly impractically) large values of the mass of the submerged body against which the floater is reacting. This may be explained by the incapacity (due to insufficient inertia of body 2) of producing the effect intended with latching: to keep the floater unmoving during part of the wave cycle and in this way (approximately) bringing the floater velocity into phase with the diffraction (or excitation) force.
It was found that reactive phase control can hardly be achieved in an effective way with a hydraulic PTO by reversing the direction of the oil flow during part of the wave cycle. This conclusion is reinforced by the results from simulations with a PTO combining a hydraulic PTO with a "negative linear spring", which indicate that the required reactive (negative spring) force largely exceeds what can be practically provided by a hydraulic PTO circuit.

\section{REFERENCES}

[1] S. A. Noren, "Plant for utilizing kinetic energy," US Patent No. 4,277,690, 1981. (Original Swedish patent No. 7808679, 1978.)

[2] L. Cleason, J. Forsberg, A. Rylander and B. O. Sjöström, "Contribution to the theory and experience of energy production and transmission from the buoy-concept," in Proceedings of the Second International Symposium on Wave Energy Utilization, Trondheim, Norway, p. 345-370, 1982.

[3] A. Weinstein, G. Fredrikson, M. J. Parks and K. Nielsen, "AquaBuOY, the offshore wave energy converter numerical modelling and optimization," in Proceedings of the MTTS/IEEE Techno-Ocean '04 Conference, Kobe, Japan, vol. 4, pp. 1854-1859, 2004.

[4] J. Falnes, "Wave-energy conversion through relative motion between two single-mode oscillating bodies," Journal of Offshore Mechanics and Arctic Engineering, vol. 121, pp. 32-38, 1999.

[5] U. A. Korde, "Phase control of floating bodies from an on-board reference," Applied Ocean Research, vol. 23, pp. 251-262, 2001.

[6] U. A. Korde, "Systems of reactively loaded coupled oscillating bodies in wave energy conversion," Applied Ocean Research, vol. 25, pp. 79-91, 2003.

[7] S. J. Beatty, B. J. Buckham and P. Wild, "Frequency response tuning for a two-body heaving wave energy converter," in Proceedings of $18^{\text {th }}$ International Offshore and Polar Engineering Conference, Vancouver, p. 342-348, 2008.

[8] J. Falnes, Ocean Waves and Oscillating Systems. Cambridge: Cambridge University Press, 2002.

[9] A. Hulme, "The wave forces acting on a floating hemisphere undergoing forced periodic oscillations," Journal of Fluid Mechanics, vol. 121, pp. 443-463, 1982.

[10] Y. Goda, Random Seas and Design of Maritime Structures. 2nd edition. Singapore: World Scientific, 2000.

[11] J. Falnes, "Optimum control of oscillation of wave-energy converters," International Journal of Offshore and Polar Engineering, vol. 12, pp. 147-155, 2002.

[12] J. Hals, T. Bjarte-Larsson and J. Falnes, "Optimum reactive control and control by latching of a wave-absorbing semisubmerged heaving sphere," in Proceedings of $21^{\text {st }}$ International Conference on Offshore Mechanics and Arctic Engineering, Oslo, Norway, 2002.

[13] K. Budal and J. Falnes, "Interacting point absorbers with controlled motion," in B. Count (editor), Power from Sea Waves. London: Academic Press, pp. 381-399, 1980.

[14] A. F. de O. Falcão, "Phase control through load control of oscillatingbody wave energy converters with hydraulic PTO system," Ocean Engineering, vol. 35, pp. 358-366, 2008.

[15] U. A. Korde, "Latching control of deep water wave energy devices using an active reference," Ocean Engineering, vol. 29, pp. 1343$1355,2002$.

[16] A. Babarit and A. H. Clément, "Optimal latching control of a wave energy device in regular and irregular waves," Applied Ocean Research, vol. 28, pp. 77-91, 2006.

[17] A. F. de O. Falcão, "Modelling and control of oscillating-body wave energy converters with hydraulic power take-off and gas accumulator," Ocean Engineering, vol. 34, pp. 2021-2032, 2007. 